\title{
Research on building the formula to determine the rate of penetration for polycrystalline diamond compact bits
}

\author{
Tu Van Truong, Hung Tien Nguyen *, Duong Hong Vu \\ Hanoi University of Mining and Geology, Hanoi, Vietnam
}

ARTICLE INFO ABSTRACT

\section{Article history:}

Received 16 ${ }^{\text {th }}$ Feb. 2021

Revised 21 ${ }^{\text {st }}$ May 2021

Accepted 19th June 2021

\section{Keywords:}

Dalamber's principle,

Drilling,

Optimize drilling parameters,

PDC bit,

Rate of penetration.

\begin{abstract}
Nowadays, polycrystalline diamond compact (PDC) drill bits are widely used in the oil and gas industry when drilling in soft rocks. However, parameters used for the PDC bit are usually based on the instructions of the drill manufacturer with a very wide adjustment range. Therefore, it is necessary to have a specific formula in order to determine the rate of penetration parameter (ROP) for the PDC bit in evaluating the influence of the parameters, rock mechanical properties and other parameters on the rate of penetration parameter (ROP). From there, it gives reasonable parameters and improves the design of the PDC bit to improve drilling efficiency. The article applies theoretical analysis method and Dalamber's principle to illuminate and build up the impact force model for PDC bits in the rock destruction process. From the impact force model, a formula to determine ROP for PDC bits was proposed. Finally, the authors applied the research results to the actual data obtained from the Nam Rong - Doi Moi oil field. The formula for determining the rate of penetration parameter (ROP) for the PDC bit that the authors have built has high accuracy and can be applied to many different rock.
\end{abstract}

Copyright (C) 2021 Hanoi University of Mining and Geology. All rights reserved.

${ }^{*}$ Corresponding author

E - mail: nguyentienhung.dk@humg.edu.vn

DOI: 10.46326/JMES.2021.62(3a).07 


\title{
Tạp chí Khoa học Kỹ thuật Mỏ - Địa chất
}

Trang điện tử: http://tapchi.humg.edu.vn

\section{Nghiên cứu xây dựng công thức xác định tốc độ cơ học khoan cho choòng PDC}

\author{
Trương Văn Từ ,Nguễn Tiến Hùng *,Vũ Hồng Dương \\ Trường Đại học Mỏ - Địa chất, Hà Nội, Việt Nam
}

THÔNG TIN BÀI BÁO

Quá trình:

Nhận bài $16 / 03 / 2021$

Sửa xong 21/5/2021

Chấp nhận đăng 19/6/2021

Tù̀ khóa:

Choòng PDC,

Khoan,

Nguyên lý Damlamber,

Tối ưu hoá thông số chế

độ khoan.

Vận tốc cơ học khoan.
TÓM TẮT

Hiện nay, choòng polycrystalline diamond compact (PDC) đước sư dung rộng rãi và phổ biến trong ngành công nghiệp khoan dầu khí khi thi công trong các địa tầng trầm tích chứa đất đá có đặc tính mềm, dẻo. Tuy nhiên, các thông số chế độ khoan sử dụng cho choòng PDC thường căn cú vào hướng dẫn của Nhà sản xuất choòng với khoảng điều chỉnh rất rộng. Vì vậy, cần có công thức cu thể để xác định tốc độ cơ học khoan đối với choòng PDC để đánh giá múc độ ảnh hưởng của các thông số chế độ khoan, tính chất co lý đá và các thông khác tới tốc độ co học khoan. Tù đó, đưa ra nhũ̃ng thông số chế độ khoan hợp lý và cải tiến thiết kế của choòng nhằm nâng cao hiệu suất khoan. Bài báo đã sử dụng các phương pháp phân tích lý thuyết, nguyên lý Dalamber nhằm phân tích các lực tác dụng lên răng choòng trong quá trình phá huỷ đất đá để xây dựng công thức xác định tốc độ co học khoan cho choòng PDC. Sau đó tiến hành xác định hê thực nghiệm dựa trên các số liệu thực tế thu được tù̀ cự mỏ Nam Rồng - Đồi Mồi. Cồng thúc xác định tốc độ co học khoan cho choòng PDC mà nhóm tác giả xây dựng được có độ chính xác cao và có thể áp dụng cho nhiều đối tượng đất đá khác nhau.

C 2021 Trường Đại học Mỏ - Địa chất. Tất cả các quyền được bảo đảm.

\section{Mở đầu}

Trong công nghiệp khoan dầu khí hiện nay, choòng PDC được sử dụng rộng rãi, phổ biến và dần thay thế toàn bộ choòng 3 chóp xoay khi khoan trong đất dá trầm tích có đặc tính mềm, dẻo do nhũng ưu điểm vượt trội mà chúng mang lại như: tốc độ khoan cơ học cao, rút ngắn thời gian

\footnotetext{
*Tác giả liên hệ

E - mail: nguyentienhung.dk@humg.edu.vn DOI: 10.46326/JMES.2021.62(3a).07
}

khoan, giảm thiểu nguy cơ phức tạp sực cố, khả năng kết hợp tốt với hệ thống lái chỉnh xiên (RSS),... Về bản chất, choòng PDC là loại choòng lưỡi cắt được trang bị các răng PDC có bề mặt được chế tạo từ kim cương đa tinh thể, được sử dụng khoan trong đất đá mềm và dẻo, dựa theo nguyên lý cắt vỡ và cho tốc độ cơ học khoan rất cao (lên đến $60 \div 70 \mathrm{~m} / \mathrm{h}$ ) (Soloviev, Nguyễn Tiến Hùng, 2015) Tuy nhiên, khi khoan trong đất đá không đồng nhất, đặc biệt là đất đá có độ cứng và độ mài mòn cao, các răng bị mòn nhanh dẫn đến tuổi thọ và năng suất của choòng PDC bị giảm mạnh (Soloviev, Nguyễn Tiến Hùng, 2015; Nguyễn 
Tiến Hùng và nnk, 2018; Nguyễn Thế Vinh và nnk, 2018; ). Thực tế cho thấy, việc sử dụng các thông số chế độ khoan đối với choòng PDC thường căn cứ vào hướng dẫn mà đơn vị sản xuất choòng cung cấp với khoảng điều chỉnh khá rộng, vì vậy trong trường hợp điều kiện khoan thực tế thay đổi, rất khó để đưa ra được thông số chế độ khoan hợp lý phù hợp với những thay đổi đó. Hiện nay, chưa có công thức cụ thể xác định tốc độ cơ học khoan đối với choòng PDC. Do đó, việc xây dựng công thức xác định tốc độ cơ học khoan cho choòng PDC mang ý nghĩa thời sự và cấp thiết, giúp xác định thông số, chế độ khoan hợp lý khi thi công trong các điều kiện khác nhau và cải tiến thiết kế choòng $\mathrm{PDC}$ nhằm tiến tới hoàn thiện chúng.

\section{Cơ sở lý thuyết và phương pháp nghiên cứu}

Các nghiên cứu về mức độ ảnh hưởng của góc lắp đặt răng choòng PDC $\alpha$ tới nguyên lý và hiệu quả phá huỷ đất đá chỉ ra rằng, đối với kiểu góc lắp đặt "âm" thì góc lắp đặt răng choòng tối ưu là $45 \div 55^{\circ}$, khi đó choòng phá huỷ đất đá theo nguyên lý cắt vỡ, tốc độ cơ học khoan đạt cao, trong khi mức độ mòn của răng choòng là thấp nhất (Bashkatov, 2010; Soloviev và nnk, 2015; Nguyễn Thế Vinh và Nguyễn Tiến Hùng, 2017).

Nhằm xây dựng công thức xác định tốc độ cơ học khoan cho choòng PDC, nghiên cứu này đã sử dụng nguyên lý Dalamber nhằm xây dựng và nghiên cứu hệ lực tác động lên răng choòng trong quá trình phá huỷ đất đá (Hình 1 ).

Chiếu hệ lực tác động lên răng choòng trên trục $0 x$, thể xác định lực tác động lên răng choòng theo phương ngang như sau:

$$
P_{o p}=F_{2}-F_{1}+N_{1} \sin \alpha
$$

Trong đó: $P_{o p}$ - lực tác động lên răng choòng theo phương ngang, $\mathrm{N} ; F_{2}$ - lực ma sát tác động lên đầu nhọn răng choòng, $F_{2}=N_{2} f ; F_{1}$ - lực ma sát tác động lên mặt phẳng đầu răng choòng, $F_{1}=$ $N_{1} f ; N_{1}$ - phản lực tác động lên mặt phẳng đầu răng choòng, $\mathrm{N} ; N_{2}$ - phản lực tác động lên đầu nhọn răng choòng, $\mathrm{N} ; f$ - hệ số ma sát giữa răng choòng và đất đá; $\alpha$ - góc lắp đặt răng choòng, độ.

Suy ra:

$$
P_{o p}=N_{2} f-N_{1} f+N_{1} \sin \alpha
$$

Chiếu hệ lực tác động lên răng choòng trên trục $\mathrm{O} z$, có thể xác định phản lực $N_{2}$ dưới dạng sau:

$$
N_{2}=P_{p}-N_{1} \cos \alpha-N_{1} \sin \alpha
$$

Trong đó: $P_{p}$ - lực dọc trục tác động lên răng choòng, $\mathrm{N}$.

Cộng phương trình (2) và (3) nhận được kết quả như sau:

$$
P_{o p}=P_{p} f+N_{1}\left(\sin \alpha-2 f \cos \alpha-f^{2} \sin \alpha\right.
$$

Trong đó: $N_{1}$ được xem là khả năng chống lại sự phá huỷ của đất đá và có thể xác định được theo công thức (Neskoromnux và Borisov, 2013):

$$
N_{1}=\sigma_{c k} A_{c k}(1+\operatorname{tg} \varphi)
$$

Trong đó: $\sigma_{c k}$ - giới hạn bền cắt của đất đá, $\mathrm{MPa} ; A_{c k}$ - diện tích cắt đất đá, $\mathrm{m}^{2}$.

Giả sử rằng, răng choòng $\mathrm{PDC}$ lắp đặt kiểu "góc âm" sẽ cắt đất đá theo hình khối omn và theo phương của lực $P_{o p}$ (Hình 1). Khối này dịch chuyển theo mặt phẳng $\mathrm{om}$ và hai mặt phẳng còn lại của khối omn, nơi mà chịu tác động của ứng suất cắt $\sigma_{c k}$.

Vì vậy, diện tích cắt đất đá được xác định là tổng diện tích của mặt cắt trên phẳng om (một nửa diện tích của hình elip có chiều dài trục là $p$ ) và hai mặt phẳng còn lại của khối omn.

$$
A_{c k}=\frac{\pi \sqrt{d_{p}} \delta_{p}^{3 / 2} \sin \alpha+2 \delta_{p}^{2} \sin \left(\alpha-\alpha_{1}\right)}{2 \sin \alpha_{1} \sin \alpha}
$$

Trong đó: $\alpha_{1}$ - góc cắt, độ; $\alpha_{1}=$ $\frac{\pi / 4-\left(\varphi+\theta_{f}-w_{c}\right)}{2} ; \varphi$ - góc nội ma sát, độ; $\theta_{f}$ - góc ma sát giữa răng choòng và một phần đất đá bị nghiền nát, $\theta_{f}=8 \div 13^{\circ}$ (Mori và Fumentro, 1994); $w_{c}$ - góc trước (Hình 1), độ; $d_{p}$ - đường kính của răng choòng (Hình 1), m; $\delta_{p}$ - độ ngập răng choòng (Hình 1), m.

Từ phương trình (5), (6) thu được như sau:

$$
\begin{gathered}
N_{1}=\sigma_{c k}(1+ \\
\operatorname{tg} \varphi) \frac{\pi \sqrt{d_{p}} \delta_{p}^{3 / 2} \sin \alpha+2 \delta_{p}^{2} \sin \left(\alpha-\alpha_{1}\right)}{2 \sin \alpha_{1} \sin \alpha}
\end{gathered}
$$

Độ ngập răng choòng $\delta_{p}$ được xác định như sau (Nguyễn Thế Vinh, Nguyễn Tiến Hùng, 2017):

$$
\delta_{p}=\left(\frac{P_{p}}{2 J \sigma_{n}(\operatorname{ctg} \alpha+\operatorname{tg} \alpha)(1+\operatorname{tg} \varphi) \sqrt{d_{p}}}\right)^{2 / 3}
$$

Trong đó: $J=(1+f \sin 2 \alpha) ; \sigma_{n}$ - giới hạn bền nén của đất đá, MPa. 
Tại đây, nghiên cứu đề xuất phương án tính tốc độ cơ học khoan theo phương pháp E.F. Epshtein (Е.Ф. Эпштейн) cho choòng lưỡi cắt (Neskoromnux, 2017; Spivak và Popov, 1994). Giả sử, răng choòng PDC lắp đặt kiểu "góc âm" có đường kính của răng là $d_{r}$ (Hình 2), số cánh của choòng là $\mathrm{m}$, mỗi cánh có số lượng răng PDC là $i$, tải trọng lên choòng là $P_{z}$, lực dọc trục tác động lên mỗi răng là $P_{r}$. Như vậy, $P_{z}=P_{r}$. m. $i$.

Với lực dọc trục $P_{r} . i$ tác động lên cánh choòng thì độ ngập cánh choòng là $\delta_{p}$, được xác định theo công thức (8). Tương ứng với đó, độ sâu khoan được trong 1 vòng quay của choòng sẽ là:

$$
h_{o}=\delta_{p} \cdot m
$$

Độ sâu khoan được trong khoảng thò̀i gian $t_{1}$ được tính theo công thức sau:

$$
h=\delta_{p} \cdot m \cdot n \cdot t_{1}
$$

Mặt khác, trong quá trình làm việc, các răng của choòng sẽ bị mòn theo thời gian. Nếu chiều cao mòn của răng là $y_{o}$ (Hình $2 \mathrm{a}$ ), thì độ ngập răng choòng theo thời gian sẽ là:

$$
\delta_{r}^{T}=\delta_{p}-y_{o}
$$

Độ sâu khoan được sau 1 vòng quay của choòng khi bị mòn sẽ là:

$$
h_{o}^{T}=\delta_{r}^{T} \cdot m
$$

Nếu độ mòn của cánh choòng sau 1 vòng quay là $y$, thì giá trị này có thể xác định được bằng phương pháp tính toán thể tích mòn của vật liệu
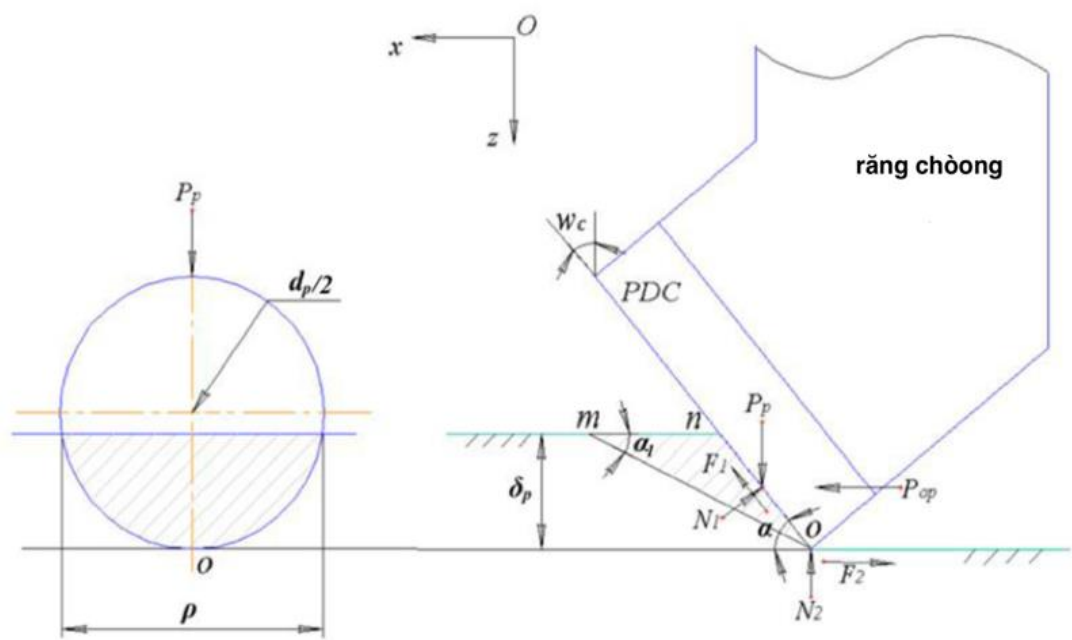

Hình 1. Hệ lực tác động lên răng choòng PDC trong quá trình phá huỷ đất đá

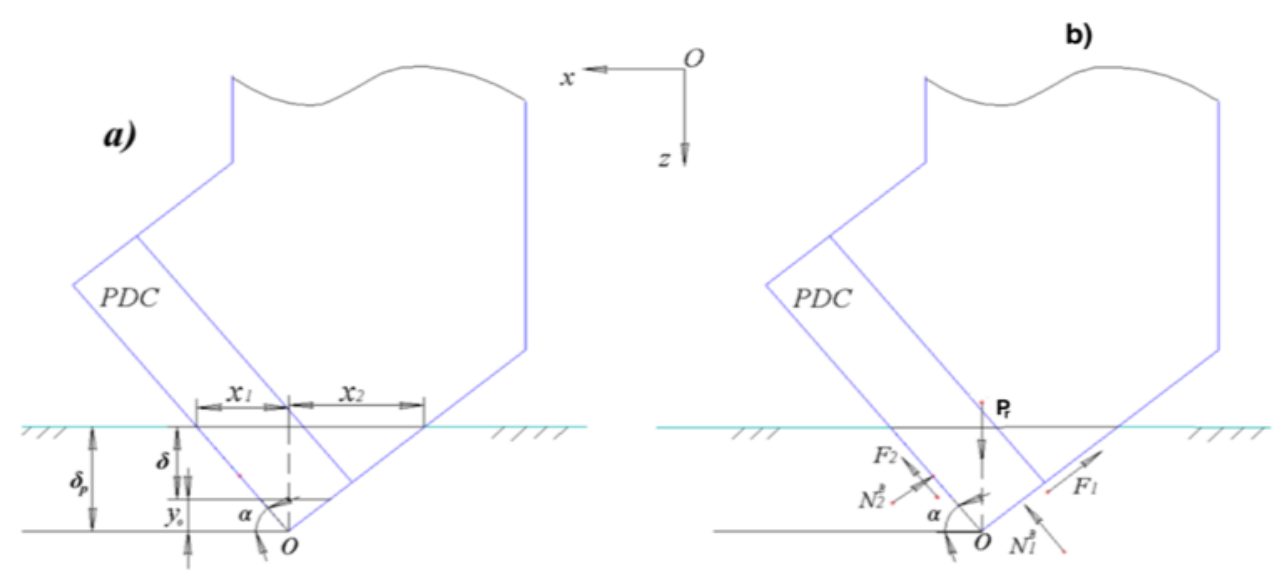

Hình 2. Mô hình quá trình cắt đất đá của một răng choòng PDC: a) độ mòn răng choòng mòn theo thòi gian; b) lực tác động lên răng choòng khi tạo ra độ ngập trong quá trình phá huỷ đất đá. 
chế tạo choòng khi nó chịu lực tác động của lực ma sát trong quá trình phá huỷ đất đá. Thể tích mòn của vật liệu chế tạo choòng sau một vòng quay được xác định như sau:

$$
V=\frac{y^{2}(\operatorname{ctg} \alpha+\operatorname{tg} \alpha) D \cdot m}{4}
$$

Mặt khác, thể tích mòn của vật liệu chế tạo choòng khoan tỉ lệ thuận với công của lực ma sát:

$$
V=k_{i} \cdot A_{1}
$$

Trong đó: $k_{i}$ - hệ số mòn thể tích của vật liệu chế tạo choòng khi chịu lực ma sát trong quá trình phá huỷ đất đá, $\frac{m^{3}}{k G . m} ; A_{1}$ - công của lực ma sát sau một vòng quay của choòng.

$$
\begin{gathered}
A_{1}=q_{i} \cdot m \cdot b \cdot f_{g} \int_{0}^{D / 2} R_{i} \cdot d R \int_{0}^{2 \pi} d \varphi \\
A_{1}=\pi m \cdot q_{i} \cdot b \cdot f_{g} \frac{D^{2}}{4}
\end{gathered}
$$

Trong đó: $q_{i}$ - áp suất tiếp xúc, Pa; $b$ - chiều rộng bề mặt chịu lực của cánh choòng, $\mathrm{m} ; f_{g}$ - hệ số ma sát của rằng choòng với đất đá; $D$ - đường kính choòng, $\mathrm{m}$.

Áp suất tiếp xúc $q_{i}$ phụ thuộc vào dạng profile của cánh choòng và ở mức độ nào đó có thể xác định được như sau:

$$
q_{i}=\frac{2 P_{z}}{m b D} \cos \alpha_{i}
$$

Như vậy:

$$
A_{1}=\frac{\pi f_{g} D P_{z} \cos \alpha_{i}}{2}
$$

Công của lực ma sát sau thời gian $t_{1}$ với tần số quay choòng $n$ sẽ là:

$$
\begin{gathered}
A=A_{1} n t_{1} \\
A=\frac{\pi n t_{1} f_{g} D P_{z} \cos \alpha_{i}}{2}
\end{gathered}
$$

Trong đó: $\alpha_{i}$ - góc tạo bởi tiếp tuyến tại một điểm bất kỳ với profile của cánh choòng và đường thẳng nằm ngang.

Như vậy, thể tích mòn của choòng được tính như sau:

$$
V=\frac{\pi k_{i} n t_{1} f_{g} D P_{z} \cos \alpha_{i}}{2}
$$

Từ hai phương trình (13) và (19) ta thu được như sau:

$$
\begin{aligned}
\frac{y^{2}(\operatorname{ctg} \alpha+\operatorname{tg} \alpha) D \cdot m}{4} & \\
= & \frac{\pi \cdot k_{i} \cdot n \cdot t_{1} \cdot f_{g} \cdot D \cdot P_{z} \cdot \cos \alpha_{i}}{2}
\end{aligned}
$$

Suy ra:

$$
y=\sqrt{\frac{2 \pi \cdot k_{i} \cdot n \cdot t_{1} \cdot f_{g} \cdot D \cdot P_{z} \cdot \cos \alpha_{i}}{(\operatorname{ctg} \alpha+\operatorname{tg} \alpha) m}}
$$

Với công thức (20), có thể tính được độ mòn của choòng tại thời điểm bất kỳ trong quá trình làm việc. Tương ứng như vậy, giá trị độ ngập răng choòng tại một thời điểm bất kỳ có thể tính được theo công thức sau:

$$
\begin{gathered}
h_{o}^{T}=\delta_{r}^{T} \cdot m-y=\delta_{p} m- \\
\sqrt{\frac{2 \pi \cdot k_{i} \cdot n \cdot t_{1} \cdot f_{g} \cdot D \cdot P_{z} \cdot \cos \alpha_{i}}{(\operatorname{ctg} \alpha+\operatorname{tg} \alpha) m}}
\end{gathered}
$$

Nhận thấy, giá trị y thay đổi theo thời gian và chiều sâu khoan được trong khoảng thời gian $t_{1}$ có thể xác định như sau:

$$
\begin{aligned}
d h & =h_{o}^{T} \cdot n \cdot d t_{1} ; d h=\delta_{p} m_{n d t_{1}}- \\
& n \sqrt{\frac{2 \pi \cdot k_{i} \cdot n \cdot f_{g} \cdot D \cdot P_{z} \cdot \cos \alpha_{i}}{(\operatorname{ctg} \alpha+\operatorname{tg} \alpha) m}} \sqrt{t_{1}} d t_{1}
\end{aligned}
$$

Tương ứng như vậy, chiều sâu khoan trong khoảng thời gian từ $t_{o}$ đến $t_{1}$ được xác định như sau:

$$
\begin{gathered}
h_{t_{o}-t_{1}}=\int_{t_{o}}^{t_{1}} h_{o}^{T} \cdot n \cdot d t_{1}=\int_{t_{o}}^{t_{1}} \delta_{p} m n d t_{1}- \\
\quad \int_{t_{o}}^{t_{1}} n \sqrt{\frac{2 \pi \cdot k_{i} \cdot n \cdot f_{g} \cdot D \cdot P_{z} \cdot \cos \alpha_{i}}{(\operatorname{ctg} \alpha+\operatorname{tg} \alpha) m}} \sqrt{t_{1}} d t_{1} \\
\frac{2}{3} n \sqrt{\frac{2 \pi \cdot k_{t} \cdot n \cdot f_{g} \cdot D \cdot P_{z} \cdot \cos \alpha_{i}}{(c t g \alpha+\operatorname{tg} \alpha) m}}\left(\sqrt{t_{1}^{3}}-\sqrt{t_{0}^{3}}\right. \\
\text { Nếu } t_{o}=0, \text { thì } \\
h_{t_{o}-t_{1}} \\
=\delta_{p} m n t_{1} \\
-\frac{2}{3} n t_{1} \sqrt{\frac{2 \pi \cdot k_{i} \cdot n \cdot t_{1} \cdot f_{g} \cdot D \cdot P_{z} \cdot \cos \alpha_{i}}{(c t g \alpha+\operatorname{tg} \alpha) m}}
\end{gathered}
$$

Mặt khác khi $h_{o}^{T}$ tiến đến 0 , khi đó $\delta_{p} m=y$ (Hình 2a):

$$
\delta_{p} m=\sqrt{\frac{2 \pi \cdot k_{i} \cdot n \cdot t_{1} \cdot f_{g} \cdot D \cdot P_{z} \cdot \cos \alpha_{i}}{(\operatorname{ctg} \alpha+\operatorname{tg} \alpha) m}}
$$


Giải phương trình (24) với ẩn là $t_{1}$ ta xác định được thời gian làm việc hiệu quả với một choòng khoan PDC:

$$
t_{n}=\frac{\delta_{p}{ }^{2} m^{3}(\operatorname{ctg} \alpha+\operatorname{tg} \alpha)}{2 \pi \cdot k_{i} \cdot n \cdot f_{g} \cdot P_{z} \cdot \cos \alpha_{i}}
$$
sẽ là:

Như vậy, tổng tiến độ choòng sau tời gian $t_{n}$

$$
\begin{gathered}
h_{t_{o}-t_{n}}=\left[\delta_{p} m n-\frac{2}{3} n \sqrt{\frac{2 \pi \cdot k_{i} \cdot n \cdot t_{1} \cdot f_{g} \cdot D \cdot P_{z} \cdot \cos \alpha_{i}}{(\operatorname{ctg} \alpha+\operatorname{tg} \alpha) m}}\right] t_{n} \\
h_{t_{o}-t_{n}}=\frac{\delta_{p}{ }^{2} m^{4}(\operatorname{ctg} \alpha+\operatorname{tg} \alpha)}{6 \pi \cdot k_{i} \cdot f_{g} \cdot P_{z} \cdot \cos \alpha_{i}} t_{n}
\end{gathered}
$$

Như vậy, tốc độ cơ học khoan trung bình có tính đến yếu tố mòn choòng xác định như sau:

$$
\begin{gathered}
V_{c h}=\frac{h_{t_{o}-t_{n}}}{t_{n}}= \\
\frac{\delta_{p}{ }^{2} m^{4}(\operatorname{ctg} \alpha+\operatorname{tg} \alpha)}{6 \pi \cdot k_{i} \cdot f_{g} \cdot P_{z} \cdot \cos \alpha_{i}} \cdot \frac{2 \pi \cdot k_{i} \cdot n \cdot f_{g} \cdot P_{z} \cdot \cos \alpha_{i}}{\delta_{p}{ }^{2} m^{3}(\operatorname{ctg} \alpha+\operatorname{tg} \alpha)} \\
V_{c h}=20 \delta_{p} m n .
\end{gathered}
$$

Thực tế tại Việt Nam cho thấy, đối với choòng PDC khoan trong đất đá trầm tích có đặc tính mềm dẻo tại địa tầng Mioxen, hầu như răng choòng không bị mòn, các tổn hao răng choòng chủ yếu là sứt mẻ và vỡ răng. Vì vậy, để kiểm chứng lại công thức, nghiên cứu này đã sử dụng công thức tính tốc độ cơ học của choòng khi các răng PDC chưa bị mòn:

$$
\begin{gathered}
V_{\text {cho }}=\delta_{p} m n(\mathrm{~m} / \mathrm{ph}) \\
V_{\text {cho }}=60 \delta_{p} \cdot m \cdot n \cdot(\mathrm{m} / \mathrm{h})
\end{gathered}
$$

Trong đó:

$$
\delta_{p}=\left(\frac{P_{p}}{2 J \sigma_{n}(\operatorname{ctg} \alpha+\operatorname{tg} \alpha)(1+\operatorname{tg} \varphi) \sqrt{d_{p}}}\right)^{2 / 3} ; n \text { - tần }
$$

số quay choòng ( $\mathrm{v} /$ phút).

\section{Kết quả và thảo luận}

Nghiên cứu này đã sử dụng công thức (29) cùng số liệu đầu vào phù hợp với điều kiện khoan các giếng tại cụm mỏ Nam Rồng - Đồi Mồi nhằm tính toán tốc độ cơ học, cụ thể như sau: $\sigma_{n}=1,2$ $\mathrm{MPa} ; \alpha=75^{\circ}$ (theo số liệu thực tế đo được); $\varphi=$ $35^{\circ} ; d_{p}=0,025 \mathrm{~m} ; f=0,1$. Sau đó, tiến hành thống kê, xử lý các số liệu thực tế của 3 giếng khoan 406 - RCDM, 404RC, 420RC thuộc cụm mỏ Nam Rồng - Đồi Mồi và thu được thông số chế độ khoan, vận tốc cơ học trung bình thực tế, hệ số thực nghiệm (Bảng 1).

Theo số liệu Bảng 1, nghiên cứu nay rút ra một số nhật xét như sau:

- Công thức xác định tốc độ cơ học khoan đối vói choòng PDC (29) cho kết quả khá chính xác với hệ số thực nghiệm $\mathrm{K}$ dao động trung bình trong khoảng $0,79 \div 0,91$;

- Mặc dù các loại choòng khoan do các hãng khác nhau sản xuất và vận hành với các thông số chế độ khoan khác nhau, tuy nhiên công thức (29) vẫn cho kết quả tương đối sát với kết quả khoan thực tế;

- Khi sử dụng tải trọng lên choòng ở mức cao (7٪11 tấn) thì vận tốc cơ học tăng đến $292,7 \%$ so vói khi sử dụng tải trọng lên choòng ở mức thấp ( 2 tấn);

- Khi tải trọng lên choòng tăng, hệ số thực nghiệm $\mathrm{K}$ tăng và có xu hướng tiến đến 1 .

\section{Kết luận và kiến nghị}

Từ những nghiên cứu bên trên có thể đưa ra một số kết luận và kiến nghị như sau:

- Công thức xác định vận tốc cơ học khoan cho choòng PDC cho kết quả có độ chính xác cao với hệ số sai số từ $0,79 \div 0,91$ đối với đối tượng nghiên cứu (Bảng 1). Đối với các đối tượng khác, để sử dụng công thức này cần tiến hành xác định hệ số thực nghiệm riêng;

- Việc xác định tải trọng lên choòng hợp lý có ý nghĩa quan trọng và quyết định đến tốc độ cơ học khoan;

- Việc xây dựng công thức xác định vận tốc cơ học khoan cho choòng PDC cho phép xác định thông số, chế độ khoan tối ưu cho từng điều kiện cụ thể;

- Hệ số thực nghiệm $\mathrm{K}$ phụ thuộc nhiều vào thông số tải trọng lên choòng, khi tăng tải trọng lên choòng thì hệ số thực nghiệm có xu hướng tăng và tiến đến 1 ;

- Đối với công tác khoan trong địa tầng Mioxen thuộc cụm mỏ Nam Rồng - Đồi Mồi nói riêng và bể Cửu Long nói chung, nên sử dụng tải trọng lên choòng lớn (>11 tấn) nhằm đạt được vận tốc cơ học khoan cao;

- Cần tiếp tục nghiên cứu và kiểm toán công thức bằng cách sử dụng nhiều hơn số liệu đầu vào và đầu ra thực tế nhằm xác định chính xác hệ số thực nghiệm của từng vùng mỏ; 
Bảng 1. Tốc độ cơ học khoan được tính theo công thức (28) và thực tếcủa các giếng khoan tại cụm mỏ Nam Rồng - Đồi Mồi

\begin{tabular}{|c|c|c|c|c|c|c|c|c|c|}
\hline \multicolumn{2}{|c|}{ Giếng khoan } & \multirow{2}{*}{ Địa tầng } & \multicolumn{2}{|c|}{ Loại choòng } & \multicolumn{2}{|c|}{$\begin{array}{c}\text { Thông số chế độ } \\
\text { khoan }\end{array}$} & \multirow{2}{*}{\begin{tabular}{|c|} 
Vận tốc \\
cơ học lý \\
thuyết \\
$(\mathrm{m} / \mathrm{h})$
\end{tabular}} & \multirow{2}{*}{$\begin{array}{l}\text { Vận tốc } \\
\text { cơ học } \\
\text { thực tế } \\
(\mathrm{m} / \mathrm{h})\end{array}$} & \multirow{2}{*}{$\begin{array}{c}\text { Hệ số } \\
\text { thực } \\
\text { nghiệm } \\
\text { K }\end{array}$} \\
\hline $\begin{array}{l}\text { Mã hiệu } \\
\text { giếng }\end{array}$ & $\begin{array}{c}\text { Khoảng } \\
\text { khoan (m) }\end{array}$ & & Mã hiệu & $\begin{array}{c}\text { Hãng sản } \\
\text { xuất }\end{array}$ & $\begin{array}{c}\mathrm{n} \\
\text { (v/phút })\end{array}$ & P (tấn) & & & \\
\hline 406 - RCDM & $2000 \div 2300$ & Mioxen & QD605X & $\begin{array}{c}\text { Baker } \\
\text { Hunges }\end{array}$ & 135 & 11,1 & 49,1 & 44,9 & 0,91 \\
\hline $404-\mathrm{RC}$ & $2000 \div 2300$ & Mioxen & QD605X & $\begin{array}{c}\text { Baker } \\
\text { Hunges }\end{array}$ & 165 & 6,9 & 63,9 & 52,7 & 0,82 \\
\hline $420-\mathrm{RC}$ & $2000 \div 2300$ & Mioxen & MRS519HBPX & Smith & 130 & 2,18 & 22,8 & 18 & 0,79 \\
\hline
\end{tabular}

- Tiếp tục nghiên cứu công thức vận tốc khoan cơ học đối với trường hợp có tính đến độ mòn của răng choòng theo thời gian (28) dựa trên số liệu thu thập thực tế về tiến độ choòng, sau đó xác định vận tốc cơ học khoan trung bình;

\section{Đóng góp của các tác giả}

Tác giả Trương Văn Từ lên kế hoạch, tiến hành thu thập, xử lý số liệu và kiểm tra tiến độ công việc. Nguyễn Tiến Hùng tiến hành phân tích lý thuyết, đưa ra mô hình lực tác động lên răng choòng PDC trong quá trình phá huỷ đất đá. Vũ Hồng Dương thu thập số liệu và chỉnh sửa nội dung.

\section{Tài liệu tham khảo}

Bashkatov D. N. (2010). Biện giải góc lắp đặt răng choòng lưỡi cắt. Tạp chí KHKT "Kỹ sư dầu khí”. 3, 9-23. Башкатов Д.Н. Обоснование угла установки резцов в долотах лопастного типа. Инженернефтяник. -No 3. -С, 9-23.

Nguyễn Thế Vinh, Nguyễn Tiến Hùng (2017). Determining the back rake angle of PDC cutters for drilling through heterogeneous rock at miocene and oligocene formations, Nam Rong - Doi Moi reservoir. Tạp chí KHKT Mỏ - Địa chất, Số 5,123 - 127.

Nguyễn Tiến Hùng , Nguyễn Thế Vinh , Doãn Thị Trâm, Nguyễn Văn Thành. (2018). Ảnh hưởng của góc lắp đặt răng tới tuổi thọ của lưỡi khoan hợp kim cứng. ERSD 2018, 32-35.

Nguyễn Thế Vinh, Nguyễn Tiến Hùng, Nguyễn Trần Tuân, Nguyễn Văn Thành. (2018). Đặc điểm mòn răng choòng khi khoan định hướng bằng hệ thống lái chỉnh xiên hoạt động theo nguyên tắc đẩy choòng. Hội nghị khoa học kỷ niệm 30 năm khai thác dầu từ đá móng Bạch Hổ. 311-317.

Neskoromnux V. V., Borisov K. I. (2013). Phân $\mathrm{t}$ ch qua tr nh cắt đất đá của răng choong PDC. Tạp chí ĐH Tổng hợp Tomsk, 1. 191-195. Нескоромных В.В., Борисов К.И (2013). Аналитическое исследование процесса резания - скалывания горнои породы долотом с резцами PDC. Известия Томского политехнического университета. Томск: Томскии политехническии университет. №1, 191 - 195.

Neskoromnux V. V. (2017). Nguyên lý phá hủy đất đá trong công tác khoan. ĐH Quoc Gia Siberia. 336 trang. Нескоромных В.В., (2017). Разрушение горных пород при бурении скважин. Сибирскии федеральныи университет. Красноярск, 336 с.

Mori V., Fumentro D. (1994). Cơ lý đất đá trong thăm dò và khai thác dầu khí. Bản dịch NXB: Hoà Binh, 195 trang. Мори В., Фурментро Д (1994). Механика горных пород применительно к проблемам разведки и добычи нефти. Перевод с французского и английского под ред. чл. - кор. РАН Н. М. Проскурякова. М.: Мир. 195 с.

Soloviev N. V., Nguyễn Tiến Hùng, (2015). Công nghệ khoan dầu khí tại các mỏ thuộc Xí nghiệp Liên doanh Việt - Nga. Tạp chí KHKT "Kỹ sư dầu khí”. Số 5. 45-49. Соловьев Н.В., Нгуен Тиен Хунг (2015). Разработка элементов эффективной технологии бурения скважин на месторождениях углеводородов 
«Вьетсовпетро». Научно - технический журнал «Инженер - нефтяник», №5. Санкт Петербург. 45 - 49.

Soloviev N. V., Arsentiev U. A., Nguyễn Tiến Hùng, (2015). Biện giải các thông số kỹ thuật của choòng lưỡi cắt. Tạp chí KHKT "Kỹ sư dầu khí". Số 5. 45-49. Соловьев Н.В., Арсентьев Ю.А., Нгуен Тиен Хунг, (2015). Теоретический метод обоснования конструктивных параметров долот режуще-скалывающего действия. Научно-технический журнал «Инженер- нефтяник». - No3. -C. 16-25.

Spivak A. I., Popov A. N., (1994). Nguyên lý phá huỷ đất đá trong khoan. NXT: Lòng Đất. 257 trang. Спивак А.И., Попов А. Н. (1994). Разрушение горных пород при бурении скважин. Недра -Москва. - 257 с. 